\title{
Molecular radiotheragnostics in prostate cancer
}

\author{
Authors: Yong $\mathrm{Du}^{\mathrm{A}}$ and Sabina Dizdarevic ${ }^{\mathrm{B}}$
}

Two different molecular radio-theragnostic principles are applied in prostate cancer, providing a personalised management for those patients. Firstly, radiopharmaceuticals with the same or similar mechanism of action but different energy (gamma- $\gamma$, eg ${ }^{99 \mathrm{~m}} \mathrm{Tc}$-diphosphonates or positron- $\beta+$, eg $18 \mathrm{~F}$ $\mathrm{NaF}$ emitting isotopes) can be used to identify patients with osteoblastic metastases for a treatment with bone seeking beta ( $\beta$-) or alpha ( $\alpha-$ ) emitting radionuclides to deliver targeted molecular radiotherapy. A number of such $\beta$ - emitting molecules have been used for bone palliation. More recently, an alpha emitting ${ }^{223} \mathrm{Ra}$-dicholoride demonstrated not only symptomatic relief but also significantly improved overall survival in castration-resistant prostate cancer with predominant bone metastases. The second principle involves utilisation of the same prostatic specific membrane antigen (PSMA) or similar compound (eg PSMA-11, PSMA-617), but different label with either $\beta+\left({ }^{68} \mathrm{Ga}\right)$ or $\gamma\left({ }^{99 \mathrm{~m}} \mathrm{Tc}\right)$ emitting radioisotope for imaging and subsequently $\beta$ - $\left({ }^{177} \mathrm{Lu}\right)$ or $\alpha\left({ }^{225} \mathrm{Ac}\right)$ emitting radionuclide for treatment.

\section{Introduction}

Prostate cancer is the most common cancer in men, affecting approximately 1 in 9 males. It is also the second most common cause of cancer death in males in the UK, accounting for approximately $13 \%$ of all cancer deaths in males. ${ }^{1}$ Almost all patients dying from prostate cancer today have castrationresistant prostate cancer (CRPC) and patients with CRPC have a $90-95 \%$ probability of developing bone metastases, which lead to severe morbidity - including bone pain, pathological fractures, spinal cord compression and haematological consequences of bone marrow infiltration. ${ }^{2-5}$

Because of the importance of bone metastases in overall disease progression and quality of life, bone targeted therapy constitutes an essential part of the treatment of CRPC. Clinically, management of bone metastases requires a multidisciplinary approach. Moderate bone pain is usually controlled by conventional analgesics, following the World Health Organization 'three step ladder'; localised pain could be treated effectively with

Authors: ${ }^{\text {A }}$ consultant in nuclear medicine and PET-CT, Royal Marsden NHS Foundation Trust, London, UK; B principle lead consultant in imaging and nuclear medicine, Brighton and Sussex University Hospitals NHS Trust and honorary reader and BSMS PETCT lead, Brighton and Sussex Medical School, Brighton, UK external beam radiotherapy. However, a substantial amount of patients experience widespread multifocal bone metastases where the above measures become less useful. ${ }^{3-6}$

Two classes of agents are specially targeting bone metastases in prostate cancer: targeting bone resorption and bone-seeking radiopharmaceuticals. Zoledronic acid is a bisphosphonate that delays skeletal-related events (SREs) by inhibiting osteoclast mediated bone resorption. Denosumab is a human monoclonal antibody against receptor activator of nuclear factor kappa-B ligand (RANKL), which has proven to be more effective than zolendronic acid for SRE prevention. These treatments, however, do not have direct tumouricidal effects and have demonstrated limited symptomatic relief. ${ }^{3-8}$ Over the past several decades, bone-seeking radiopharmaceuticals have demonstrated their value in diagnosing and treating painful bone metastases.

In addition, a new radiolabelled theragnostic compound targeting prostate-specific membrane antigen (PSMA) has been developed to select patients and deliver irradiation to all tumour sites, including osseous, nodal and visceral metastases.

\section{Radiotheragnostic pathway for metastatic bone disease}

The patient selection process for targeted bone therapy follows a theragnostic pathway, which involves the same theragnostic

\section{Key points}

Prostate cancer is the most common cancer in men, leading to approximately $13 \%$ of cancer deaths in men

Bone is the most common metastatic site from prostate cancer

Bone-seeking radionuclides have been used in the diagnosis of bone metastases, for selecting appropriate patients and in bone pain palliation

A new bone-seeking radionuclide, ${ }^{223} \mathrm{Ra}$, has demonstrated survival benefit in addition to symptomatic relief

PSMA appears to be a very promising target for molecular radiotheragnostics using radiopharmaceuticals like ${ }^{68} \mathrm{Ga} /{ }^{177}$ Lu-PSMA

KEYWORDS: ${ }^{223} \mathrm{Ra},{ }^{89} \mathrm{Sr}$, Bone metastases, prostate cancer, PSMA, radionuclide 


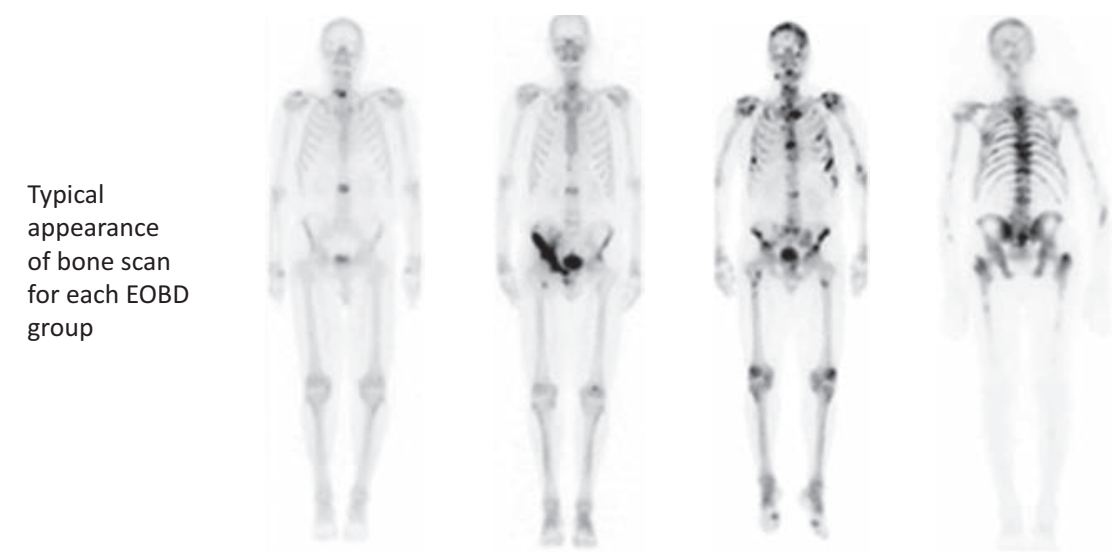

\begin{tabular}{|l|c|c|c|c|}
\hline EOBD group & 1 & 2 & 3 & 4 \\
\hline Number of metastases & $<6$ & $6-20$ & $>20$ & Superscan \\
\hline
\end{tabular}

agent for diagnosis and therapy, but using different isotopes for diagnosis (emitting $\gamma / \beta+$ energy by bone scintigraphy (Fig 1)) and for therapy (emitting $\beta-/ \alpha$ ).

Given systemically, $\beta$-/ $\alpha$ radiopharmaceuticals deliver targeted irradiation to the active bony turnover sites where the metastatic infiltration and destruction is happening. Advantages of this approach include the ability to simultaneously treat multiple sites of disease, the ease of administration, the repeatability and the potential for integration or combination with other treatments. Early investigations were focused mostly on $\beta$-emitting radiopharmaceuticals such as strontium $-89\left({ }^{89} \mathrm{Sr}\right)$ chloride, samarium-153 $\left({ }^{153} \mathrm{Sm}\right)$ lexidronam and renium-186 $\left({ }^{186} \mathrm{Re}\right)$ etidronate. More recently, an alpha emitter - radium-223 $\left({ }^{223} \mathrm{Ra}\right)$ dichloride - was approved to treat bone metastases from prostate cancer. The physical characteristics of these currently approved bone-seeking radiopharmaceuticals are summarised in Table 1.

\section{Beta-emitting radiopharmaceuticals}

The efficacy of bone-seeking radiopharmaceuticals relies on their selective uptake and prolonged retention at sites of increased osteoblastic activity. Biologically, they mimic calcium deposit at sites of increased osteoblastic activity, which is secondary to tumour-related bony destruction and subsequently increased bony turnover process. These sites can be readily detected on pre-treatment bone scintigraphy as increased tracer uptake (Fig 1$)^{1,6}$ and the extent of bone disease can be assessed by Soloway score. ${ }^{9}$

The path length of the beta ray irradiation from these radiopharmaceuticals ranges $4-7 \mathrm{~mm}$. Although the radiation is concentrated at the tumour sites, adjacent bone marrow would also be affected by cross-fire irradiation and thus the dose-limiting toxicity is bone marrow suppression. A careful patient selection should be performed before treatment with bone-seeking radiopharmaceuticals, with consideration of haematological function, bone disease assessment and bone marrow involvement at pre-therapy bone scintigraphy, performance status, recent use of myelosuppressive therapies and life expectancy. Poor renal function reduces the plasma clearance of beta-emitting bone-seeking radiopharmaceuticals and could lead to higher whole-body dose and increased risk of myelotoxicity. Although the safety and toxicity of treatment in patients with renal insufficiency has not been thoroughly investigated, caution should be taken in treating such patients.

Overall, non-randomised clinical trials performed mostly in the 1970s and 1980s have demonstrated substantial bone pain palliation using ${ }^{89} \mathrm{Sr}$-chloride, ${ }^{153} \mathrm{Sm}$-lexidronam and ${ }^{186} \mathrm{Re}$-etidronate with pain relief being achieved in $60-80 \%$ of patients. ${ }^{1,2,8}$ Although these treatments have gained more acceptance in routine oncological practice, radiopharmaceutical or molecular radiotherapy remain infrequently used treatment modalities mostly because of their relatively limited availability, especially in smaller hospitals with no existing nuclear medicine expertise.

\section{${ }^{223} \mathrm{Ra}$-dichloride, an alpha particle emitting radiopharmaceutical}

${ }^{223} \mathrm{Ra}$ emission has an extremely short path length $(<100 \mu \mathrm{m})$ but high linear energy transfer (Table 1). Biologically, ${ }^{223} \mathrm{Ra}$ is similar to the beta emitting radiopharmaceuticals: it selectively concentrates in bone with increased osteoblastic activity and its progeny are retained in the bone matrix. While posing

Table 1. Physical characteristics of therapeutic radionuclides for prostate cancer

$\begin{array}{lllll}\text { Radionuclide } & \text { Half-life } & \begin{array}{l}\text { Maximum } \\ \text { energy }\end{array} & \begin{array}{l}\text { Mean } \\ \text { energy }\end{array} & \begin{array}{l}\text { Maximum } \\ \text { range }\end{array} \\ { }^{89} \mathrm{Sr} & 50.5 \text { days } & 1.4 \mathrm{MeV}(\beta) & \begin{array}{l}0.583 \\ \mathrm{MeV}(\beta)\end{array} & 7 \mathrm{~mm} \\ { }^{186} \mathrm{Re} & 3.7 \text { days } & 1.07 \mathrm{MeV}(\beta) & \begin{array}{l}0.362 \\ \mathrm{MeV}(\beta)\end{array} & 5 \mathrm{~mm} \\ & 1.9 \text { days } & 0.81 \mathrm{MeV}(\beta) & 0.229 & 4 \mathrm{~mm} \\ { }^{153} \mathrm{Sm} & 11.4 \text { days } & \begin{array}{l}5.78 \mathrm{MeV} \\ (\alpha) \text { (average) }\end{array} & & <100 \mu \mathrm{m} \\ & & (\beta) & \end{array}$

${ }^{89} \mathrm{Sr},{ }^{153} \mathrm{SM}$ and ${ }^{223} \mathrm{Ra}$ are approved for clinical use both in Europe and the USA; ${ }^{186} \mathrm{Re}$ is approved in some European countries only. 
very high tumouricidal efficacy within its short path length, it generates much more localised radiation zones, reducing exposure of surrounding normal tissue compared with beta emitters and thus has less myelotoxicity. ${ }^{223} \mathrm{Ra}$ also has a preferable physical half-life of 11.4 days and is mostly excreted through faeces. Such characteristics make it an ideal candidate to treat bone metastases in prostate cancer patients often also presenting with urinary symptoms.

${ }^{223} \mathrm{Ra}$ was brought into clinical development in 2001. A phase I clinical trial involving 31 patients with bone metastases from breast and prostate cancer demonstrated only modest and reversible haematological toxicity, which was more pronounced in the higher dose groups, while dose limiting haematotoxicity was not observed. Transient grade 1-2 diarrhoea was the most frequent adverse event.

A subsequent phase II study evaluated the efficacy of various doses given at 6 week intervals in patients with bone metastases from CRPC and demonstrated a dose-dependent effect, which was well tolerated even at the highest dose level. In a further placebo-controlled randomised phase II trial, 64 patients with painful bone metastases were randomised to receive either ${ }^{223} \mathrm{Ra}(55 \mathrm{kBq} / \mathrm{kg}$, four cycles with a 4 -weekly interval) or placebo (saline) after palliative external beam radiotherapy. The study showed a significant improvement in serum bone turnover biomarker (alkaline phosphatase), delayed PSA progression and improved overall survival. These successes led to the ALSYMPCA phase III trial, which recruited 921 patients who were randomised $2: 1$ to $55 \mathrm{kBq} / \mathrm{kg}^{223} \mathrm{Ra}$ or placebo. The trial showed significant improvements in survival: patients in the ${ }^{223} \mathrm{Ra}$ group had longer median survival than those in the placebo group (14.0 months versus 11.2 months; $\mathrm{p}=0.002$ ).

There was also significant prolonged time to first skeletal-related event (13.6 months versus 8.4 months; $\mathrm{p}=0.00046)$. $^{3,10-13}$

In summary, ${ }^{223} \mathrm{R}$ a was the first bone-targeting alpha emitter to demonstrate improved survival in CRPC patients with symptomatic bone metastases; it delayed SREs in this group of patients, improved quality of life, and showed anti-tumour efficacy and palliation of bone pain. It was approved in 2013 in both Europe and the USA and recommended by the National Institute for Health and Care Excellence for the treatment of patients with symptomatic bone metastases from CRPC and no known visceral metastases. $3,12,14,15$

\section{Theragnostic PSMA targeting prostate cancer}

Despite major advances in CRPC treatment, overall survival remains short and the search for new treatments continues. Recently, a new radiolabelled theragnostic compound targeting PSMA for both diagnostic and therapeutic applications entered this field.

PMSA is found on most prostate cancer cells and its overexpression correlates to adverse factors. Binding of a ligand leads to internalisation and retention within the cell. Lutetium-177 $\left({ }^{177} \mathrm{Lu}\right)$ is a beta emitter with a path length of approximately $2 \mathrm{~mm}$ in tissue and a preferable half-life of 6.73 days. ${ }^{177} \mathrm{Lu}$ has been used successfully as a component of radiopharmaceutical ${ }^{177} \mathrm{Lu}$-DOTATATE in treating neuroendocrine tumours. The related radio-conjugation technologies have been well developed. In the last few years, several studies have observed excellent in vivo tumour targeting
Fig $2 .{ }^{68}$ Ga-PSMA-PET/CT scan demonstrates multiple nodal and bony metastases in a patient with MCRPC. A - MIP; $\mathrm{B}$ - fused PET/CT image; C corresponding $\mathrm{CT}$ image of the pelvis on bone window. PSMA avid bony infiltration involving much of the right ischium is arrowed. The patient could be suitable for ${ }^{177}$ Lu-PSMA targeted molecular radiotherapy. $\mathrm{CT}=$ computerised tomography; $\mathrm{mCRPC}=$ metastatic castration-resistant prostate cancer; MIP = maximum intensity projection; $\mathrm{PET}=$ positron emission tomography; PSMA = prostatic specific membrane antigen
A

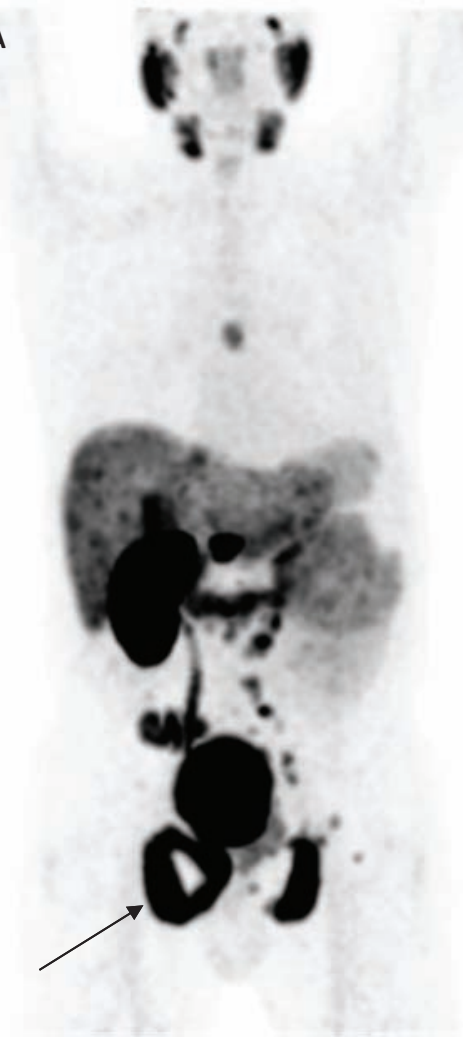

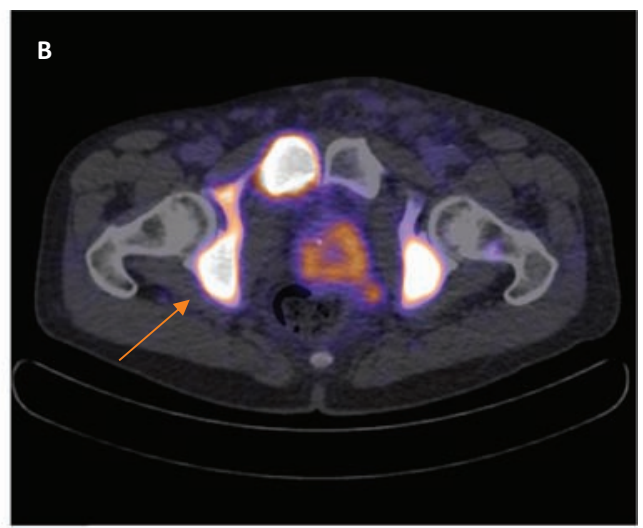

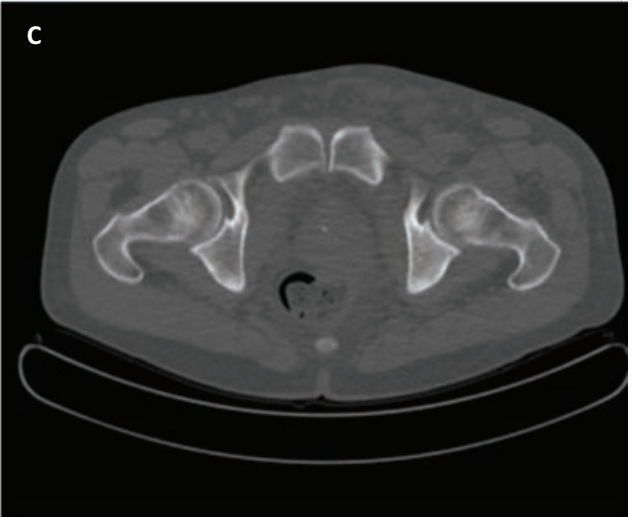


using ${ }^{177} \mathrm{Lu}$-PSMA-617 and produced a promising response rate of $40-70 \%$ with only mild toxicity in patients with metastatic CRPC who had failed chemotherapy. ${ }^{16-20}$

Clinically, the patient selection process for ${ }^{177} \mathrm{Lu}-\mathrm{PSMA}$ therapy follows the typical theragnostic pathway: patients would undergo a gallium-68 $\left({ }^{68} \mathrm{Ga}\right)$-PSMA PET/CT scan first to test and confirm the presence of PSMA expression on tumour cells ( ${ }^{68} \mathrm{Ga}$ is a positron emitter used for PET/CT scanning); if the scan confirms satisfactory PSMA expression as illustrated in Fig 2, ${ }^{177} \mathrm{Lu}$-PSMA would be given for therapy. Different from the bone-seeking radiopharmaceuticals, PSMA is expressed on tumour cells within and outside the bone and thus ${ }^{177} \mathrm{Lu}$-PSMA could deliver systemic targeted irradiation to all tumour sites.

In addition, comparison studies have showed a better diagnostic performance with ${ }^{68} \mathrm{Ga}$-PSMA PET/CT than the other widely used tracer choline-PET/CT, especially in localising metastatic prostate cancer. ${ }^{21-24}$ Although ${ }^{68}$ Ga-PSMA PET/CT imaging and subsequent ${ }^{177}$ Lu-PSMA remain experimental, early clinical observations have been very promising. ${ }^{16,20,25}$

More recently, early studies indicated an alpha emitter, actinium-225 labelled PSMA, might be more effective than ${ }^{177} \mathrm{Lu}-\mathrm{PSMA}$ in treating metastatic CRPC although no randomised comparison has been conducted. ${ }^{26}$

\section{Conclusions}

Bone-seeking radiopharmaceuticals have been used to select patients for targeted molecular radiotherapy. They are also playing an increasingly instrumental role in the treatment of patients with CRPC, as exampled by the recent advent of ${ }^{223}$ Ra-dichloride. Excitingly, early studies suggest that newer molecular theragnostic approaches, such as using PSMA radioligands, could potentially provide even more effective treatment options for this devastating disease.

\section{Conflicts of interest}

The authors have no conflicts of interest to declare.

\section{References}

1 Finlay IG, Mason MD, Shelley M. Radioisotopes for the palliation of metastatic bone cancer: a systematic review. Lancet Oncol 2005;6:392-400.

2 Bodei L, Lam M, Chiesa C et al. EANM procedure guideline for treatment of refractory metastatic bone pain. Eur J Nucl Med Mol Imaging 2008;35:1934-40.

3 Parker C, Nilsson S, Heinrich D et al. Alpha emitter radium-223 and survival in metastatic prostate cancer. N Engl J Med 2013;369:213-23.

4 Coleman RE, Guise TA, Lipton A et al. Advancing treatment for metastatic bone cancer: consensus recommendations from the Second Cambridge Conference. Clin Cancer Res 2008;14:6387-95.

5 Coleman RE, Lipton A, Roodman GD et al. Metastasis and bone loss: advancing treatment and prevention. Cancer Treat Rev 2010;36:615-20.

6 Lewington VJ. Bone-seeking radionuclides for therapy. J Nucl Med 2005;46(Suppl 1):38S-47S.

7 Paes FM, Serafini AN. Systemic metabolic radiopharmaceutical therapy in the treatment of metastatic bone pain. Semin Nucl Med 2010;40:89-104.

8 Paes FM, Ernani V, Hosein P, Serafini AN. Radiopharmaceuticals: when and how to use them to treat metastatic bone pain. J Support Oncol 2011;9:197-205.

9 Soloway MS, Hardeman SW, Hickey D et al. Stratification of patients with metastatic prostate cancer based on extent of disease on initial bone scan. Cancer 1988;61:195-202.
10 Sartor O, Coleman R, Nilsson S et al. Effect of radium-223 dichloride on symptomatic skeletal events in patients with castration-resistant prostate cancer and bone metastases: results from a phase 3, doubleblind, randomised trial. Lancet Oncol 2014;15:738-46.

11 Vogelzang NJ, Coleman RE, Michalski JM et al. Hematologic safety of radium-223 dichloride: baseline prognostic factors associated with myelosuppression in the ALSYMPCA trial. Clin Genitourin Cancer 2017;15:42-52.e8.

12 Nilsson S, Franzen L, Parker C et al. Bone-targeted radium-223 in symptomatic, hormone-refractory prostate cancer: a randomised, multicentre, placebo-controlled phase II study. Lancet Oncol 2007;8:587-94.

13 Parker C, Finkelstein SE, Michalski JM et al. Efficacy and safety of radium-223 dichloride in symptomatic castration-resistant prostate cancer patients with or without baseline opioid use from the phase 3 ALSYMPCA trial. Eur Urol 2016;70:875-83.

14 Pandit-Taskar N, Larson SM, Carrasquillo JA. Bone-seeking radiopharmaceuticals for treatment of osseous metastases, Part 1: alpha therapy with 223Ra-dichloride. J Nucl Med 2014;55:268-74.

15 Umeweni N, Knight H, McVeigh G. NICE guidance on radium-223 dichloride for hormone-relapsed prostate cancer with bone metastases. Lancet Oncol 2016;17:275-76.

16 Afshar-Oromieh A, Babich JW, Kratochwil C et al. The rise of PSMA ligands for diagnosis and therapy of prostate cancer. J Nucl Med 2016;57(Suppl 3):79S-89S.

17 Barrio M, Fendler WP, Czernin J, Herrmann K. Prostate specific membrane antigen (PSMA) ligands for diagnosis and therapy of prostate cancer. Expert Rev Mol Diagn 2016;16:1177-88.

18 Heck MM, Retz M, D’Alessandria C et al. Systemic Radioligand therapy with (177)Lu labeled prostate specific membrane antigen ligand for imaging and therapy in patients with metastatic castration resistant prostate cancer. J Urol 2016;196:382-91.

19 Kratochwil C, Afshar-Oromieh A, Kopka K, Haberkorn U, Giesel FL. Current status of prostate-specific membrane antigen targeting in nuclear medicine: clinical translation of chelator containing prostate-specific membrane antigen ligands into diagnostics and therapy for prostate cancer. Semin Nucl Med 2016;46:405-18.

20 Scarpa L, Buxbaum S, Kendler D et al. The 68Ga/177Lu theragnostic concept in PSMA targeting of castration-resistant prostate cancer: correlation of SUVmax values and absorbed dose estimates. Eur J Nucl Med Mol Imaging 2017;44:788-800.

21 Schwenck J, Rempp H, Reischl G et al. Comparison of 68Ga-labelled PSMA-11 and 11C-choline in the detection of prostate cancer metastases by PET/CT. Eur J Nucl Med Mol Imaging 2017;44:92-101.

22 Morigi JJ, Stricker PD, van Leeuwen PJ et al. Prospective comparison of 18F-fluoromethylcholine versus 68Ga-PSMA PET/CT in Prostate cancer patients who have rising PSA after curative treatment and are being considered for targeted therapy. J Nucl Med 2015;56:1185-90.

23 Yu CY, Desai B, Ji L, Groshen S, Jadvar H. Comparative performance of PET tracers in biochemical recurrence of prostate cancer: a critical analysis of literature. Am J Nucl Med Mol Imaging 2014;4:580-601.

24 Afshar-Oromieh A, Zechmann CM, Malcher A et al. Comparison of PET imaging with a (68) Ga-labelled PSMA ligand and (18) F-choline-based PET/CT for the diagnosis of recurrent prostate cancer. Eur J Nucl Med Mol Imaging 2014;41:11-20.

25 Rahbar K, Ahmadzadehfar H, Kratochwil C et al. German multicenter study investigating 177Lu-PSMA-617 radioligand therapy in advanced prostate cancer patients. J Nucl Med 2017;58:85-90.

26 Kratochwil C, Bruchertseifer F, Giesel FL et al. 225Ac-PSMA-617 for PSMA-targeted alpha-radiation therapy of metastatic castrationresistant prostate cancer. J Nucl Med 2016;57:1941-4.

Address for correspondence: Dr Yong Du, Department of Nuclear Medicine, The Royal Marsden NHS Foundation Trust, Downs Road, Sutton SM2 5PT, UK.

Email:yong.du@rmh.nhs.uk 